\title{
Value of measurement of maternal serum alpha fetoprotien in diagnosis of pathologically adherent placenta in cases of placenta pravia
}

Emad Ahmed Fyala.

Lecturer of Obstetric and

Gynecology

Department of Obstetrics

and Gynaecology, Faculty of

Medicine, Mansoura University,
Emad Ahmed Fyala.

Lecturer of Obstetric and Gynecology

Department of Obstetrics and Gynaecology, Faculty of Medicine, Mansoura University, Tel: 01005623409

E mail; emadfyala@yahoo.com

\section{$\underline{\text { Abstract }}$}

Objective: to investigate the significance of elevated MSAFP in diagnosing pathologically adherent placenta among women with placenta previa and its predictive value for pregnancy outcome.

Study Design: A retrospective study involved eighty women with placenta praevia divided into 4 equal groups according the degree of invasion as suspected by TAS, Doppler and MRI studies and confirmed postoperatively by histo-pathological examination. G1; included those with placenta pravia alone G2; those with placenta accreta, G3; those with increta degree while G4 contained those with placenta percreta. MSAFP level were evaluated for all patients between 34-36 weeks gestational age.

Results: Baseline characteristics of the study group showed no significant difference among groups 1, 2, 3 as regard maternal age, gravidity, parity, fetal gestational age and body mass index $(\mathrm{p}>0.05)$ but when compared separately with group 4 , there was significant increased mean $(+\mathrm{SD})(\mathrm{P}<0.05)$ but not in the body mass index. Maternal risk factors of placenta praevia and abnormal placental adherence proved more previous uterine scars in groups 3 and $4,(\mathrm{P}<$ 0.002). MSAFP levels represented a remarkable increase in groups 2 and 3 and shooting up in group 4 (mean+SD, 91.3+19.1, 153.2+38.1, $178.3+25.2,263.3+36.1$ respectively, $P$ value 0.001 ). Fetal and maternal outcomes as well as intraoperative findings showed some statistical significant differences between the studied groups as maternal intraoperative blood loss was found more evident in G4 (mean + SD 931 \pm 312$)$ compared to G1, 2 and $3(640 \pm 191,767 \pm 331$, $834 \pm 284$; respectively, P value 0.02 ) with more liability for postpartum hemorrhage, 7 cases $(35 \%)$ versus 0,2 and 3 cases in G1, 2, 3 . respectively (P 0.003).IIAL was resorted to in 7 cases in G4 compared to nothing in G1 and G2 while in 2 cases only in G3. Bladder injuries were reported in 5 cases in G4 and 1 case in G3 but not in $\mathrm{G} 1$ and 2 (P 0.002). The mean operative time in minutes (SD) was found relatively shorter in G1 but longer in G2 and 3 but longest in G4 (34 $\pm 9.1,44 \pm 5.1,49 \pm 9.2,55 \pm 8.1$, respectively, $\mathrm{P}$ value 0.01$)$. There were more need for intra and postoperative blood transfusion in cases of G4, 7 cases versus 2 cases only in G2 and 3. Again, there was more requested emergent caesarean hysterectomy in G4 (4 cases versus one case in G3, P 0.01). The postoperative data confirmed that there was evident longer duration of hospital stay in G4 $(5+$ 2.3) compared to other groups 1,2 and $3(3.2+1.1,3+1.2,3.5+1.3$, $P$ value 0.01$)$. Admission to intensive care unit was recorded higher 
in G4, 5 cases compared to 1 case only in G3 (P $0.002)$. No maternal mortality recorded as well as neonatal intensive care unit admission.ROC analyses finds elevated MSAFP stated the maximum sum of sensitivity and specificity for the significant test and showed the AUC was $0.752(95 \% \mathrm{CI}$ : $0.505-0.979)$ with $83.54 \%$ sensitivity and $71.53 \%$ specificity $(\mathrm{p}=0.042)$.

Conclusion: Increased third trimester MSAFP levels can predict morbidly adherent placenta in placenta previa patients, with no other obvious cause, as well as intraoperative complications.

Key words: placenta previa, morbid adherence, alpha-fetoprotein.

\section{Introduction}

The morbidly adherent placenta is an actual increasing concern of the child bearing age and is estimated in climbing rate parallel to the increasing rate of cesarean deliveries[1]. The morbid placental adherence is defined as complete or partial invasion of the chorionic villi into the myometrium up to the peritoneal covering[2]. Recently; there is much improvements in the imaging techniques, mainly high-resolution ultrasound and magnetic resonance imaging, but this situation still constitute a significant diagnostic challenge for the obstetricians and health care providers[3]. Various possible risk factors and predictive markers were been extensively investigated regarding this issue. Studies have proven that important risk factors included prior cesarean deliveries, placenta previa, prior uterine surgery or curettage, advanced maternal age, and multiparity $[4,5]$. On the other hand, entities examined for predicting abnormal invasion of placenta were the maternal serum biological markers in first and second trimesters namely, elevated maternal serum alpha-fetoprotein (MSAFP), human chorionic gonadotropin (hCG) and pregnancy-associated plasma protein-A (PAPP-A) [6-9].

The morbid adherence of the placenta is almost always carrying significant risks for both pregnant women and their babies. Common encountered maternal threats include mainly excessive hemorrhages, disseminated intravascular coagulopathy, urinary tract and bowel injuries, more need for emergent hysterectomy, massive blood and blood products transfusion and therefore more increased risk of maternal deaths[10]. The adverse outcomes also will catch the neonates being more liable for a number of complications due to unscheduled preterm delivery $[10,11]$. From this aspect, there should be an intense need for early diagnosis [12] and preparing proper strategies to enhance the antenatal care and management in tertiary care centers by multidisciplinary approach and well equipped experienced team $[3,11]$.

The author aimed to evaluate the significance of estimating the level of maternal serum alpha-fetoprotein in predicting degree of placental adherence to give a signal for those who will be referred early to be managed in a tertiary referral hospital.

\section{Patients and methods}

A retrospective comparative study conducted at Gynecology and Obstetrics Departments, Mansoura University Hospitals from Feb 2016 through October 2017. Our study was approved by the local research ethical committee at Mansoura University Hospitals and College of Medicine Institutional research board $(\mathrm{R} / 17.01 .76)$. The research steps were conducted in accordance to ethical principles for medical researches involving human subjects of the world medical association (WMA; Declaration of Helsinki) as revised during the 95thWMA general assembly held in Seoul, South Korea, October 2008. All patients with placenta praevia admitted in the high risk pregnancy unit for elective cesarean deliveries were recruited for sharing in the research at a gestational age 34-36 weeks. Eighty participates only were included in the research and divided into 4 equal groups: group [1]were those with placenta pravia without any suspicion of chorionic invasion or accretion as evidenced by trans-abdominal sonography (TAS), Doppler studies or MRI, whereas the other three groups were clustered according the suspected degree of invasion by the same investigations; group [2] involved those with evident placenta accreta, group [3] included those with proved placenta inccreta meanwhile group [4]contained cases with percreta degree. All patients were verbally consented and then each participant gave a written consent before to be included. Patients with fetal congenital anomalies, multiple pregnancy and maternal diseases suspecting elevated serum alpha fetoprotein levels (e.g. current or history of ovarian 
tumors with pregnancy) were excluded from our study. Additionally, pregnant women with gestational age before 34 weeks were not involved as such patients may be missed or dropped out before admission while taken from antenatal care unit and also at this late gestational age placental localization is almost always confirmed. Situations that might give any misleading results of MSAFP were not also involved e.g. pregnancies achieved by artificial reproductive technology, any known systemic disease, cigarette and alcohol consumption and patients on chronic medication, placental chorioangioma or evident abruption.

The medical records for our participants were collected including demographic and anthropometric data, obstetric, medical and family history and any risk factor suspecting the occurrence of praevia. Complete general and obstetric examinations as well as the main laboratory investigation mainly complete blood count, coagulation profile and renal and liver function tests were obtained.

All patients had undergone trans-abdominal ultrasound to show the type of placenta previa or any criteria suggestive of invasion depending on presence of one or more of the following; myometrial thickness less than $1 \mathrm{~mm}$, large intra-placental blood lakes, defined as placental lacunae by color Doppler ultrasound enhancement, loss of the retro-placental sonolucent zone, thinning or disruption of the hyper-echoic serosa-bladder interface and presence of focal exophytic masses invading the urinary bladder [13-15].

Ultrasound was done for all patients with one apparatus by the same sonographer physician using Logic p-52012 device with 3.5-5 MHz curvilinear transducer (for gray scale and color Doppler techniques) while the patients lying supine with medium-full urinary bladder.

Thereafter a venous blood sample was collected from every participant, centrifuged and analyzed immediately using an enzyme-linked immune-sorbent assay (ELISA) and measuring its level by automated biochemical analyzer using DEIFIA Xpress System (Perkin Elmer Life and Analytical Sciences, Waltham MA, USA). The MSAFP cutoff value of 2-MoM was used to provide the best combination of sensitivity and specificity for the out-come levels [16]. All participants were delivered by elective cesarean section after competing 37 weeks or when maternal or fetal distress emerges. All operations were performed under spinal anesthesia by the same senior obstetrician. The uterus was repaired with continuous unlocked suture in 2 layers using Vicryl 1 suture (Johnson \& Johnson, USA). The peritoneum was left un-sutured while the muscle layer was opposed with Vicryl 0 . The rectus sheath was closed by Vicreyl 1, and the skin was then closed with sub-cutical suture by Proline double zero in all groups of patients. The operative findings were examined carefully for the type and localization of the placenta, degree of invasion in the uterine wall and the near-by organs. The degree of pathological adherence was confirmed by biopsy taken from the uterine wall at the placental bed. Cases with morbid placental adherence requiring hysterectomy were confirmed according to the previously established criteria of the placenta adherence involving all layers of the uterine walls[17]. Other intraoperative complications, e.g. bleeding, ureteric or bladder injuries were also recorded. The number of packed red blood cells, fresh frozen plasma and other blood products transfused were then obtained from postoperative records. All maternal and fetal outcomes were evaluated including the data of admission for both in the intensive care units. All collected data were recorded at Medline data base and subjected for statistical analysis.

Data were collected, tabulated and statistically analyzed by using the Statistical Package for the Social Sciences version 17 (SPSS, Chicago, IL). Chi- square test was used to compare the association between categorical variables of all groups and Fisher exact test was used where necessary. Student t-test was used to compare means of quantitative variables in parametric data. The optimal cut-off-points of laboratory measurements to distinguish cases with and without placental adherence were evaluated by Receiver Operating Characteristic (ROC) analyses calculating area under the curve (AUC) as giving the maximum sum of sensitivity and specificity. $\mathrm{P}$ value $<0.05$ was set significant. 


\section{$\underline{\text { Results }}$}

A total number of 80 patients diagnosed as placenta praevia, 60 of whomhad a variable degree of intra-myometrial invasion, at 34-36 weeks were involved in our study. They were divided into 4 equal groups.

Baseline characteristics of the four groups are shown in table [1]; despite there was no significant difference between groups 1, 2, 3 as regard maternal age, gravidity, parity, fetal gestational age and body mass index ( $p>0.05)$. but when comparing each group separately with group 4 , there was significant increased mean $(+\mathrm{SD})$ in the maternal age, gravidity as well as parity $(\mathrm{P}<0.05)$ but not in the body mass index. Maternal risk factors of placenta praevia and abnormal placental adherence was also studied in table [1]. Studied risk factors, mainly previous uterine scars were found in all groups by varying degrees but 3 or more previous uterine scars were more evident in groups 3 and 4,8 cases $(40 \%)$ and , 12 cases $(60 \%)$ respectively $(\mathrm{P}<0.002)$, table [1].Maternal serum alpha-fetoprotein level in all groups was represented also in table (1)and despite it is found near normal in group 1 but it is remarkably increased in groups 2 and 3 and shooting up in group 4 (mean (SD) is, $91.3+19.1,153.2+38.1,178.3+25.2,263.3+36.1$ respectively and $P$ value 0.001 ). The minimum value recorded was $32 \mathrm{ng} / \mathrm{ml}$ in G1 whereas the highest value recorded in $\mathrm{G} 4$ was $387 \mathrm{ng} / \mathrm{ml}$.

Fetal and maternal outcomes as well as intraoperative findings showed some statistical significant differences between the studied groups as shown in table [2]. Maternal intraoperative blood loss was found more evident in G4 (mean + SD 931 \pm 312) compared to G!, 2 and $3(640 \pm 191,767 \pm$ $331,834 \pm 284$; respectively, $\mathrm{P}$ value 0.02 ) with more liability for postpartum hemorrhage, 7 cases (35\%) versus nothing, 2 and 3 cases in groups 1, 2, 3 . respectively (P 0.003). Internal iliac artery ligation was resorted to in 7 cases of placenta percreta group compared to nothing in placenta pravia and accreta groups while in 2 cases only in increta patients. Bladder injuries was reported in 5 cases in G4 and 1 case in G3 but not recorded in G1 and 2 (P 0.002). the mean operative time in minutes (SD) was found relatively shorter in G1 but longer in G2 and 3 but longest in G4 (34 $\pm 9.1,44 \pm 5.1,49$ $\pm 9.2,55 \pm 8.1$, respectively, $\mathrm{P}$ value 0.01$)$. There were more need for intra and postoperative blood transfusion in cases of G4, 7 cases versus 2 cases only in G2 and 3. Again, there was more requested emergent caesarean hysterectomy in G4 (4 cases versus one case in $\mathrm{G} 3, \mathrm{P} 0.01$ ). When women in all groups were compared regarding the postoperative data, there was evident longer duration of hospital stay in G4 $(5+2.3)$ as compared to other groups 1 , 2 and $3(3.2+1.1,3+1.2,3.5+1.3, \mathrm{P}$ value 0.01$)$. Moreover, admission of cases to intensive care unit was recorded higher in G4, 5 cases compared to 1 case only in G3 (P 0.002) as shown in table [2]. No maternal mortality recorded as well as neonatal intensive care unit admission.

\section{Discussion}

The main finding of this retrospective study confirmed elevated level of MSAFP with pathologically adherent placenta estimated in late pregnancy. This elevation is significantly found directly related to the degree of invasion. To the best of our knowledge; no studies reported in our locality to investigate the relation-ship between elevated MSAFP with placental adherence of varying degrees and its reliability for use as predictor for pregnancy outcome as regarding bladder injury, PPH and caesarean hysterectomy in these patients.

Biochemically; AFP is a glycoprotein containing $\alpha$-globulin (591 amino acids) and a carbohydrate fraction existing in monomeric form with a molecular weight of $67 \mathrm{kDa}[18,19]$. It is the major serum binding protein in the fetus and playing an essential role in carrying hormones, fatty acids, bilirubin and minerals at the maternal-fetal interface [19]. It is formed early by the yolk, meanwhile later on fetal gastrointestinal tract and liver will takes place to be the main source throughout the rest of the pregnancy. It is excreted via fetal urine to be transported to the maternal serum through the placenta/ fetal membranes diffusion[20, 21]. The normal median MSAFP concentration differs according to on locality and ethnicity of the studied groups with interquartile range from $104.2-380 \mathrm{ng} / \mathrm{mL}$ [22]. From this aspect we selected our cases with exclusion of all fetal, maternal and placental causes increasing MSAFP. This comes in agreement with the opinion of some other authors [23-25].The elevated levels of MSAFP in cases of pathologically adherent placenta could be explained by a 
disruption of the maternal-fetal-placental barrier, placental vascular damage, feto-maternal bleeding or relative placental ischemia [23, 24].

Despite many authors studied and proved elevated level of MSAFP and its association with morbid placental adherence among women with placenta previa[6, 9, 25] and some used it as a predictor for pregnancy outcome, but they used it earlier in the second trimester. Our results come consistent to this finding but we investigated our patients in the third trimester owing to authorize admission of participants and avoiding dropping ones during the research time. Really this time selected is considered as one of major drawbacks of our work if we are going to use MSAFP as a predictor for placental adherence in high risk patients of placenta praevia or its pregnancy outcome, the fact which verified before by some [7, 25].Again, the association between elevation of this biological marker and emergent cesarean hysterectomy is evident and stated in our results ( 4 cases in G4 and 1 case in G3) and this comes in accordance with the results published by Gomaa, et al. [22] and Efser et al [25] but comes contrary to earlier findings by Butler et al [27] who stated no significant increase in emergent peripartum hysterectomy in cases of morbid placenta invasion despite more mothers' liability for bleeding, preterm labor and difficult placental removal. Also, in this respect, regarding adverse pregnancy outcomes, our results demonstrates consistent findings with some previous studies with an increased need of blood and blood products transfusion, ICU admission and also operative morbidities with adjacent organ injuries, mainly the urinary bladder and postoperative bleeding with consequent more hospital stay days $[11,25,27,28]$. Therefore, here we can state that successful management of morbidly adherent placenta depends mainly on an accurate preoperative diagnosis and subsequent delivery of the patient in a tertiary care center by an experienced team to avoid or minimize the possible complications. Moreover, some suggest the clinical significance of even mildly elevated MSAFP levels should be considered in the prediction of these risky obstetric situations $[7,5]$.

On evaluating the optimal cut-off point for elevated MSAFP for the prediction of morbid placenta adherence and pregnancy outcome by ROC anal- yses to state the maximum sum of sensitivity and specificity for the significant test. ROC curve of our results showed the AUC was 0.752 (95\% CI: $0.505-0.979$ )with $83.54 \%$ sensitivity and $71.53 \%$ specificity $(p=0.042)$. This comes in accord with some previous results proved recently [25].Here; the author can state that one of the best predictors for morbid placental adherence and also associated increased risk of cesarean hysterectomy, postoperative long hospital stay, adjacent organ injuries and poor pregnancy outcome $(p<0.05$ in all).

Despite our results seem promising for earlier preparation of a multidisciplinary team for managing patients with morbid placental adherence but there are few major limitations and confusing issues. These include, the retrospective design of the present study and the limited number of participants in each group and also being one center study. A prospective earlier investigations and multicenter research on a big scale of patients is recommended for more convenient and informative results.

\section{Conclusion}

Elevated MSAFP can predict intraoperative complications of morbidly adherent placenta, caesarean hysterectomy, bladder injury, PPH, admission to ICU and this strongly necessitates special precautions before surgical intervention and preparation for delivery.

\section{References}

1. 1.S. Wu, M. Kocherginsky, J.U. Hibbard. Abnormal placentation: 20-year analysis. Am J ObstetGynecol, 192 (2005), pp. 1458-1461. ArticlePDF (174KB)

2. Committee o n Obstetric Practice. Committee opinion No. 529: placenta accreta. American College of Obstetricians and Gynecologists. Obstet. Gynecol, 120 (2012), pp. 207-211[3]

3. B.H. Lim, J.M. Palacios-Jaraquemada. The morbidly adherent placenta-a continuing diagnostic and management challenge. BJOG, 122 (2015), p. 1673

4. A. Gagnon, R.D. Wilson, F. Audibert, V.M. Allen, C. Blight, J.A. Brock, et al. .Society of Obstetricians and Gynaecologists of Canada Genetics Committee. Obstetrical complications associated with abnormal maternal serum markers analytes. J Obstet. Gynaecol Can, 30 (2008), pp. 918-949 
5. Publications Committee Sfm-Fm, M.A. Belfort. Placenta accreta. Am J Obstet. Gynecol, 203 (2010), pp. 430-439

6. T.H. Hung, W.Y. Shau, C.C. Hsieh, T.H. Chiu, J.J. Hsu, T.T. High Risk factors for placenta accreta. Obstet. Gynecol, 93 (1999), pp. 545550. ArticlePDF (154KB)

7. S. Dreux, L.J. Salomon, F. Muller, F. Goffinet, J.F. Oury, ABA Study Group, L. Sentilhes. Second-trimester maternal serum markers and placenta accreta. Prenat Diagn, 32 (2012), pp. 1010-1012

8. N. Desai, D. Krantz, A. Roman, A. Fleischer, S. Boulis, B. Rochelson. Elevated first trimester PAPP-A is associated with increased risk of placenta accreta. Prenat Diagn, 34 (2014), pp. 159-162

9. D.J. Lyell, A.M. Faucett, R.J. Baer, Y.J. Blumenfeld, M.L. Druzin, Y.Y. El-Sayed, et al. .Maternal serum markers, characteristics and morbidly adherent placenta in women with previa. J Perinatol, 35 (2015), pp. 570-574.

10. R.M. Silver. Placenta accreta: we can do better! BJOG, 123 (8) (2016), p. 1356

11. Z.S. Bowman, T.A. Manuck, A.G. Eller, M. Simons, R.M. Silver. Risk factors for unscheduled delivery in patients with placenta accreta. Am J Obstet. Gynecol, 210 (2014) 241.e1-6

12. M. Sakinci, O. Kuru, M. Tosun, A. Karagoz, H. Celik, F.D. Bildircin, et al. .Clinical analysis of emergency peripartum hysterectomies in a tertiary center. ClinExpObstet. Gynecol, 41 (2014), pp. 654-658

13. Pilloni E., Alemanno M., Gaglioti P., et al. Accuracy of ultrasound in antenatal diagnosis of placental attachment disorders. Ultrasound in Obstetrics \& Gynecology. 2016; 47:302-307.

14. Rheinboldt M., Delproposto Z. Sonography of placental abnormalities: a pictorial review. Emergency Radiology. 2015; 22:401-408

15. Shih J.C., Palacios Jaraquemada J.M., SU Y.N., et al.: Role of three-dimensional power Doppler in the antenatal diagnosis of placenta accreta: Comparison with grey-scale and color Doppler techniques. Ultrasound Obstet. Gynecol., 33 (2): 193-203, 2009.

16. Ollendorff D.A., Goldberg J.M., Abu-Jawdeh G.M. and Lurain J.R.: Markedly elevated maternal serum alpha-fetoprotein associated with a normal fetus and choriocarcinoma of the placenta. Obstet. Gynecol., 16 (3 Pt. 2): 494-497, 1990.

17. H. Fox. Placenta accreta 1945-1969. ObstetGynecolSurv, 27 (1972), pp. 475-490

18. P. Pucci, R. Siciliano, A. Malorni, G. Marino, M.F. Tecce, C. Ceccarini, et al.Human alpha-fetoprotein primary structure: a mass spectrometric study. Biochemistry, 30 (1991), pp. 5061-5066

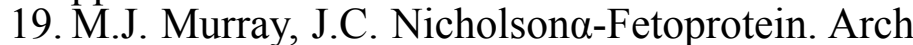
Dis Child EducPract Ed, 96 (2011), pp. 141-147

20. D. Gitlin, A. Perricelli, G.M. Gitlin. Synthesis of alpha-fetoprotein by liver, yolk sac, and gastrointestinal tract of the human conceptus. Cancer Res, 32 (1972), pp. 979-982

21. F.J. Los, H.W. De Bruijn, T. van BeekCalkoenCarpay, H.J. Huisjes. AFP transport across the fetal membranes in the human. PrenatDiagn, 5 (1985), pp. 277-281

22. MOSTAFA F. GOMAA, M.D.; AHMED E. ALBEHOUTY, M.D.; AMGAD S. ABU GAMRA, M.D.; HESHAM M. FATHY, M.D. and MAHMOUD GHALEB, M.Sc. Maternal Serum Alpha Fetoprotein Versus Ultrasound Criteria in Prediction of Outcome in Pregnancies with Suspected Morbid Adherence of the Placenta. Med. J. Cairo Univ., Vol. 82, No. 1, December: 731-735, 2014 www. medicaljournalofcairouniversity.net

23. M. Salafia, L. Silberman, N.E. Herrera, M.J. Mahoney. Placental pathology at term associated with elevated midtrimester maternal serum $\alpha$-fetoprotein concentration.Am J ObstetGynecol, 158 (1988), pp. 1064-1066. ArticlePDF (256KB)

24. C.Y. Spong, A. Ghidini, C.N. Walker, M. Ossandon, J.C. Pezzullo. Elevated maternal serum mid-trimester alpha-fetoprotein levels are associated with fetoplacental ischemia. Am J ObstetGynecol, 177 (1997), pp. 1085-1087. ArticlePDF (276KB)

25. EfserOztas, SibelOzler, Ali TurhanCaglar, AykanYucel. Analysis of first and second trimester maternal serum analytes for the prediction of morbidly adherent placenta requiring hysterectomy. Kaohsiung Journal of Medical Sciences (2016) 32, 579e585

26. BUTLER E.L., DASHE J.S. and RAMUS R.M.: Association between maternal serum alphafetoprotein and adverse outcomes in pregnancies with placenta previa. Obstet. Gynecol., 97 (1): 35-38, 2001.

27. H. Alchalabi, I. Lataifeh, B. Obeidat, F. Zayed, Y.S. Khader, N. Obeidat Morbidly adherent placenta previa in current practice: prediction and maternal morbidity in a series of 23 women who underwent hysterectomy. J Matern Fetal Neonatal Med, 27 (2014), pp. 1734-1737.

28. A. Abuhamad. Morbidly adherent placenta. SeminPerinatol, 37 (2013), pp. 359-364. ArticlePDF (3MB) 
Table [1]:Baseline characteristics and MSAFP levels of all the studied groups.

\begin{tabular}{lccccc}
\hline & G1 $(\mathbf{n}=\mathbf{2 0})$ & $\mathbf{G 2}(\mathbf{n}=\mathbf{2 0})$ & $\mathbf{G 3}(\mathbf{n}=\mathbf{2 0})$ & $\mathbf{G 4}(\mathbf{n}=\mathbf{2 0})$ & P value \\
\hline Age (years) & $28.4 \pm 4.1$ & $29.1 \pm 5.1$ & $29.1 \pm 4.2$ & $32 \pm 3.2$ & $0.03^{*}$ \\
\hline Gravidity & 4.12 & $4.3 \pm 4$ & $5.1 \pm 1.3$ & $6.2 \pm 2.1$ & $0.003^{*}$ \\
\hline Parity & $3.2 \pm 2.1$ & $3.5 \pm 2.9$ & $4.1 \pm 1.1$ & $5.1 \pm 1.2$ & $0.002^{*}$ \\
\hline BMI & $24 \pm 0.9$ & $24 \pm 0.2$ & $25 \pm 0.5$ & $25 \pm 0.2$ & 0.324 \\
\hline Risk factors: & & & & \\
Less than 2 scars & $8(40 \%)$ & $14(70 \%)$ & $12(60 \%)$ & $8(40 \%)$ & $0.002^{*}$ \\
More than 2 scars & $2(10 \%)$ & $6(30 \%)$ & $8(40 \%)$ & $12(60 \%)$ & \\
\hline GA at delivery & $38.2 \pm 0.3$ & $38.3 \pm 0.7$ & $37.1 \pm 0.4$ & $36.1 \pm 0.8$ & 0.04 \\
\hline MSAFP & $91.3 \pm 19.1$ & $153.2 \pm 38.1$ & $178.3 \pm 25.2$ & $263.3 \pm 36.1$ & 0.001 \\
\hline
\end{tabular}

Data are presented by mean $\pm \mathrm{SD}$, or percentages. $\mathrm{P}$ value was set significant when $<0.05$.

Abbreviations:BMI; body mass index, GA; gestational age, MSAFP; maternal serum alpha fetoprotein.

Table [2]:operative and postoperative findings in all the studied groups.

\begin{tabular}{lccccc}
\hline & G1 $(\mathbf{n}=\mathbf{2 0})$ & $\mathbf{G 2}(\mathbf{n}=\mathbf{2 0})$ & $\mathbf{G 3}(\mathbf{n}=\mathbf{2 0})$ & $\mathbf{G 4}(\mathbf{n}=\mathbf{2 0})$ & P value \\
\hline Mean blood loss & $640 \pm 191$ & $767 \pm 331$ & $834 \pm 284$ & $931 \pm 312$ & 0.02 \\
\hline PPH & - & $2(10 \%)$ & $3(15 \%)$ & $7(35 \%)$ & 0.003 \\
\hline IIAL & - & - & $2(10 \%)$ & $7(35 \%)$ & 0.002 \\
\hline Bladder injuries & - & - & $1(5 \%)$ & $5(25 \%)$ & 0.002 \\
\hline MOT (minutes) & $34 \pm 9.1$ & $44 \pm 5.1$ & $49 \pm 9.2$ & $55 \pm 8.1$ & 0.01 \\
\hline Blood transfusion & - & $2(10 \%)$ & $2(10 \%)$ & $7(35 \%)$ & 0.03 \\
\hline Cesarean hysterectomy & - & - & $1(5 \%)$ & $4(20 \%)$ & 0.01 \\
\hline HSD & $3.2 \pm 1.1$ & $3 \pm 1.2$ & $3.5 \pm 1.3$ & $5 \pm 2.3$ & 0.01 \\
\hline MICUA & - & - & $1(5 \%)$ & $5(25 \%)$ & 0.001 \\
\hline NICUA & - & - & - & - & - \\
\hline
\end{tabular}

Values are presented as mean $\pm \mathrm{SD}$, number (percentage), $\mathrm{p}$ value was set significant when $<0.05$.

Abbreviations: PPH, postpartum hemorrhage, IIAL; internal iliac artery ligation, HSD; hospital stay days, MICUA; maternal intensive care unit admission, NICUA; neonatal intensive care unit admission.

Table [3]:Association between degree of pathologically adherent placenta and serum level of MSAFP in patients $(\mathrm{n}=80)$.

\begin{tabular}{|c|c|c|c|}
\hline P value & $\begin{array}{c}\text { Subjects with elevated } \\
\text { MSAFP }\end{array}$ & $\begin{array}{c}\text { Patients with normal } \\
\text { level of MSAFP }\end{array}$ & Variable \\
\hline 0.561 & $2(2 / 20=3.6 \%)$ & $18(5 / 20=25 \%)$ & Placenta praevia \\
\hline 0.000 & $10(10 / 20=50 \%)$ & $10(10 / 20=50 \%)$ & Placenta accrete \\
\hline 0.046 & $12(12 / 20=60 \%)$ & $8(8 / 20=40 \%)$ & Placenta increta \\
\hline 0.04 & $14(14 / 20=70 \%)$ & $6(6 / 20=30 \%)$ & Placenta percreta \\
\hline
\end{tabular}

